\title{
Cryptic lineages respond differently to coral bleaching
}

\author{
Matias Gómez-Corrales ${ }^{1}$ and Carlos Prada ${ }^{1}$ \\ ${ }^{1}$ University of Rhode Island
}

May 19, 2020

\begin{abstract}
Coral reefs are losing coral cover across the globe largely as a result of a rise in seawater temperatures that trigger coral bleaching and induce coral mortality. How coral reefs will respond to climate change will be a function of genetic variation and how it is partitioned among species. A critical initial step is to accurately delineate species and quantify their physiological potential to cope with heat stress. Cryptic species, morphologically indistinguishable but genetically different ones, typically harbor distinct physiological variation and respond differently to climatic changes. A dominant Caribbean reef builder severely affected by climate change is the mountainous star coral, Orbicella faveolata. Recently, Dziedzic et al. (2019) reported genetic variation in the physiological response to thermal stress in a single population of this species, suggesting that variation within populations will allow these corals to adapt to rising ocean temperatures. We reanalyzed their data and found multiple cryptic lineages rather than a single panmictic population, with only one of the lineages being heat-tolerant. Our finding of hidden lineages within a threatened species highlights the varying extinction risks faced by these independently evolving groups, especially when the prospects of survival under warmer oceans seem favorable for a few of them only.
\end{abstract}

\section{Hosted file}

Gomez\&Prada_MolEcol_2020_4.pdf available at https://authorea.com/users/323714/articles/ 452266-cryptic-lineages-respond-differently-to-coral-bleaching 